action very feeble; extremities cold; breathing difficult and oppressed; the bowels had acted twice freely; no purging; perfectly sensible, but, in fact, in a state of complete collapse. The account that I collected was, that she had been taken ill the previous evening about seven, with faintness, vomiting, and pain referred to the right side, for which she took some brandy-and-water; that she with great difficulty, at a later period in the evening, dragged herself up to bed, and that she continued to get worse up to the time that I was called to her. I ascertained further, that she had had a male companion in the house with her on the Sunday afternoon, and that they had made themselves tolerably comfortable together. The last time she took her medicine containing the wine of colchicum, was at three P.M. on the Sunday. On being called te her, the only course of treatment left for me to pursue was that of supporting her with cordials and other remedies applicable to the state of collapse in which $I$ found her, with half a drachm of tincture of opium to stop the vomiting. The last ceased about ten A.M.; in every other respect she continued to get worse, the symptoms becoming aggravated until three P.M., when she died, about twenty hours after being attacked. The opinion that I gave at the time was, that some vessel had given way in the abdomen, but from what source the internal hæmorrhage had arisen $\mathcal{I}$ was utterly unable to predict.

On first seeing her in the state of collapse, it occurred to me that she might possibly be labouring under the effects of the small dose of wine of colchicum, but there was none of the purging which ordinarily accompanies the poisonous action of that medicine. Had she taken poison of any kind ? The pain and vomiting might induce the belief, but such was not my impression. Of course, under these circumstances, I was particularly anxious to obtain a post-mortem examination, the result of which I now forward to you. For a patholo gical and microscopical description, as also for perfect diagrams of the appearances, I am indebted to my friend, Dr. W. Pettigrew, who kindly offered to conduct the examination of the parts under the field of a microscope, and the consequence of which has been a much clearer and more accurate account than could otherwise have been recorded.

Post-mortem examination. External appearances: body well formed; no emaciation; fat, of the thickness of an inch, over the parietes of the abdomen, on opening into which fluid blood immediately poured out, and on laying it completely open, the whole cavity was found filled with dark fluid blood and a large quantity of coagula, the whole amounting, as near as I could ascertain, to about three quarts; it nearly filled a large chamber-vessel. Having very carefully sponged out the abdomen, I proceeded to ascertain the source whence the hæmorrhage had proceeded. The liver, spleen, kidneys, abdominal vessels, large and small, were all carefully examined, but no rupture or abrasion of any kind could be found -in fact, the abdominal viscera were all perfectly healthy and normal. On proceeding to inspect the pelvic cavity, I observed a coagulum in the left Fallopian tube. I then at once removed the uterus with its appendages, and the case became clearly developed. The uterus was slightly enlarged; the os and canal leading to the cavity were filled with a muco-sanguineous fluid. The cavity of the uterus was lined by a deciduum, by which, as usual, the orifices of the Fallopian tubes were hidden. All the appendages and ligaments appeared much congested. The right ovarium was scarred all over, from the escape of ova; and upon making an incision through the ovarium a well-marked corpus luteum was found. Upon the left side a small tumour, of the size of an almond, presented itself in the Fallopian tube, about an inch from the uterus; and corresponding to the upper part of this tumour a small rent was seen, with some very small pieces of coagulated blood about the edges. An incision being made through the tumour showed it to be composed of coagulated blood, in the centre of which was a small sac, but so much compressed by the coagulum as to render it (although dissected under water, and with a powerful glass) impossible to state, to a certainty, that it was the ovum; the appearance was much more distinct when the tumour was first cut into, and I have not the slightest doubt that it was an ovum, especially when coupled with the certainty of the deciduum being already found in the uterus. The left ovary was enlarged to the size of a small apple, and hollow. Upon the upper and back part of this was a rent, about a quarter of an inch in length, with portions also of coagulated blood about its lips; and it was from this rupture that the extensive hremorrhage had taken place. The deciduum, under the microscope, showed the usual appearances. The muco-sanguineous fluid in the canal of the uterus contained mucus and blood-globules, and also fibrin. There were no traces of spermatozoa either about the os uteri, or amongst the vaginal fluid, or with the epithelium.

The above case is very remarkable. I have searched through various works on midwifery, but have not been successful in finding one where death has occurred from the rupture and hæmorrhage of an ovarian sac, though there are, I believe, cases on record, in which death has ensued from the bursting of the Fallopian tubes. The case is one showing the absolute necessity and duty of insisting upon a postmortem examination in all sudden deaths, and without which the medical attendant is liable to incur the risk of mischievous rumours, as it is in nowise improbable that reports, in this instance, might have spread, that the patient had died from the effects of the medicine, or from poison.

Brompton square, March, 1848.

\section{ON THE THEORY OF SPASMO-PARALYSIS IN INFANTS AND ADULTS.}

Bx MARSHALL HALL, M.D., F.R.S., \&c., London.

Paralysis may depend on the exclusion of the influence either of the cerebrum; or of the spinal marrow,-that is, of both cerebrum and spinal marrow. Spasm can only arise from irritation of some part of the spinal system; but this irritation may affect the incident excitor nerves, the spinal centre, or the muscular nerves. Spasmo-paralysis is a term which I have adopted to express the varied combinations of spasm and paralysis which occur so frequently in practice.

How shall we find our way through this labyrinth ?

It may, indeed, be said that the diseases of the nervous system constitute one-third part of medicine, and that their accurate diagnosis was impossible previous to the distinction of the three divisions of that system from each other. Now this distinction depended, in its turn, on the detection and separation of one of these-the reflex spinal system-from the other two,-viz., the cerebral and ganglionic; and this again, in its turn, affords the diagnostic of the diseases of the entire nervous system.

Such is the important practical object to which I wish to devote myself, while $I$ leave the mean and ignoble caviller at the threshold of this true temple of medical science.

Infants are often born with distortion of the foot or feet, and during growth a paralytic weakness and atrophy are conjoined with the spasmodic action of the deforming muscle. A similar effect issometimes seen to take place during infancy. I have once seen a case in an adult in which the tendo Achillis became so drawn that the patient could no longer put the heel to the ground. In some cases of hemiplegia, spasmodic contraction of the hand and arm accompanies the paralytic attack. In other cases, a spasmodic contraction of the hand gradually takes place more remotely after the attack. I have seen various cases of paralysis, or spasm, distinctly, and of the two either variously combined or following each other. Now what is the just rationale-what the theory of these spasmoparalytic cases?

These questions could not be answered before the appearance of the remarkable work of M. Flourens, or before the detection of the reflex spinal system. The due limitation of the excitor and in-excitor portions of the nervous system, which we owe to $M$. Flourens, and the just appreciation of the incident and reflex forms of action, of their excito-motor powers, which has flowed from my own investigations, are the essential preliminaries to an investigation of the theory of spasm, of paralysis, and of spasmo-paralysis. To this very day $I$ observe these things confounded together, and that eren by teachers of our pupils and critics of our literature.

\section{Intra-uterine Spasmo-paralysis.}

How interesting and how valuable would be a series of accurate cases and post-mortem examinations of the various congenital spasmodic and spasmo-paralytic affections! of cheirismus, and especially of podrismus, in the varied forms, or rather deformities, of club-foot.

Is the cause of this calamity always of centric origin ? or is it sometimes the reflex action of external cold, injury, \&c.?

The class of intra-uterine diseases still requires renowed investigation, and no part of it more than affectlons of the nervous system.

Effusion over the hemispheres, and at the base of the encephalon, and along the spinal canal, is too frequently the cause of irritation-pressure or counter-pressure-on the spinal system, that division of the nervous system endowed 
with the excito-motor power. This irritation is the source of various congenital convulsive or spasmodic affections; it may be the cause of strabismas, laryngismus, \&c., of the various distortions of the hands, and especially of the feet. In the case of two brothers, similarly affected, the tendo Achillis was permanently. contracted with spasmo-paralysis of both legs; on the death of one, aged twelve, effusion on the cerebral hemispheres, at the base of the brain, and along the spinal canal, was found in considerable quantity; the arachnoid was thickened, and, over the lateral portion of one hemisphere, converted into a thin layer of bone.

\section{of Spasmo-paralysis in Infants and Children.}

Spasmo-paralysis in infants and children is of centric and of ex-centric origin - the prognosis of the former being, of course, far more formidable than that of the latter.

Teething, ahd gastric and intestinal irritation, and, I suspect, exposure of the naked surface to the cold, are the causes of the reflex or ex-centric forms of this malady. From such causes I have seen hemiplegia of the arm, or of the leg, or of both; and the proof that the affection was of reflex origin was a very happy one--viz., speedy recovery.

The event, however, is not always so fortunate.

Sometimes both legs are affected, and this affection is sometimes more observed in one leg than in the other; sometimes the spasm, sometimes the paralysis, predominates; and sometimes one leg is affected with paralysis, whilst the other is affected with spasmo-paralysis.

Spasmo-paralysis in the Adult.

But of all the cases which have come under my observation, none has been more replete with interest and anxiety than spasmo-tparalysis ocourring in the adult period of human life.

It is well known that the epileptic convulsion sometimes leaves one arm, one leg, or one side, paraly tic or hemiplegic, in: a greater or less degree. If the seizures were not to be repeated, I imagine this paralysis would frequently subside, being the effect of shock, and of the common cause or causes of the convulsion and of the hemiplegia, which is therefore not permanent. But if the shock be repeated, the paralysis may be permanent, although the convulsion subsides.

In one most interesting case, a lady, aged thirty-five, was seized with violent convulsion of the left side of the face, and of the left arm, the leg being unaffected; when the convulsion ceased, the face and arm were left extremely, if not perfectly paralytic. A degree of amendment took place; but the convulsions returned, occupying the same seats as before, and, on ceasing, again left the face, arm, and hand, absolutely paralytic.

This lady had once had phlegmasia dolens after parturition, and this leg again became swollen. But the cause of the attack of convulsions seemed to be discovered in the condition of the intestines; for these convulsions were relieved by purgatiye medicines, but were excited if those medicines acted too violently.

From the paralysis left by this serious attack or repetition of attacks, the patient recovered completely,-an additional proof that the affection had, like many cases of epileptic seizure, arisen from some cause ex-centric to the encephalon or spinal marrow. And how invaluable is this fact, in reference both to our prognosis and treatment!

Indeed, I may here observe, that spasmo-paralysis is in every respect a disease of less hopeless character than pure paralysis, inasmuch as the irritation of an organ is a less severe affection than its destruction. The diagnosis or detection of the cause is the first great object of the physician, and especially the determination of the question-Whether that cause be seated centrically or ex-centrically.

In one case, which occurred in a member of our own profession, after repeated threatenings supposed to be apoplectic, severe spasmo-paralysis supervened, and remained permanent. Bleeding had been resorted to constantly as the preventive. It ought, I believe, to have been decided, but not too severe, antacid aperients, with a strict attention to the diet, which should not have been of a mere vegetable, but of a light and digestible character.

There was, I believe, more of the epileptic than of the apoplectic in those threatenings. Is there any physical lesion? Is the case, or was the case, one admitting of recovery? How deeply interesting are all these questions?

It is plain that the new topic-new because now viewed distinctly-of spasmo-paralysis, will assume an important position amongst the objects of the physician's studies.

I have two patients under my care, at this time, with po- drismus, occurring at the ages, in one, of twenty-five, in the other, of forty-five. Both are females. In the first, the right foot is drawn upwards and inwards, and so severely as to induce great tenderness and swelling of the outer ankle. Various symptoms of nervous origin are conjoined with this deformity of the foot. In the other, the tendo Achillis in each leg is tense, and the toe only, and not the foot, much less the heel, can be put to the ground. In this ease almost every article of food or medicine is rejected by vomiting.

I do not believe that either of these cases is hysteria. There is no other symptom of hysteric character, and the temperament in both patients is staid and sedate.

\section{Conctusion.}

From the recent progress of the physiology of the nervous system, we are now enabled to conclude-

1. That paralysis, pure paralysis, may be an affection cither of the cerebrum, the spinal marrow, or the nerves; but

2. That sposm must be an affection of some part of the true spinal system; and

3. That spasmo-paralysis must at least involve in it an affection of the true spinal system, either primarily or secondarily.

There is only one exception to this last rule: it is the case of severe hemiplegia, in which, from the mere facts of the severing of the influence of volition, and the normal or physiological action of the spinal marrow-the source at once of the irritability of muscular fibre and of tone-the affected hand frequently becomes spasmodically flexed.

Here I conclude this brief paper. I think I have clearly shown in it, once more, how important, how essential, physiology is to the physician, and pointed out a distiuction to be carefully drawn between paralysis, and spasm, and spasmoparalysis, as at once a guide to our prognosis and our treats. ment.

\section{STRANGULATED UMBILICAL HERNIA SUC- CESSFULLY OPERATED UPON AFTER THE PLAN PROPOSED BY MR. GAY.}

\section{By GEO. BORLASE CHILDS, Esq., F.R.C.S.E., London.}

Mr. Chrnos was requested by Mr. Clarke, of Queen-street, to see.Mrs. T_- on the 8th of March, a very corpulent person, aged thirty-five, affected with a large umbilical hernia, which had become strangulated in the course of the previons night. Mrs. T- had been the subject of a hernial protru: sion for two years, which, although irreducible, had given hor. no inconvenience. On the occurrence of strangulation, the parts in the vicinity, as well as the contents of the hernial sac, became very tender, the abdomen tense and unyielding, which symptoms were speedily followed by a constant sense of nausea and vomiting.

The hernial tumour, at least eight inches in its longest diameter, lay principally on the abdominal parietes to the left of the umbilicus, which it depressed to a depth that made the distance between them and the integuments very considerable; and obviously consisted of a large mass of omentum as well as: intestine.

An operation for its relief was immediately required, and on determining upon the plan, a preference was given to that recently proposed by Mr. Gay, on the grounds, that from its great size, the corpulency of the patient, and the extent of wound necessary for its performance in the ordinary way, hardly any but a fatal result could be anticipated from it.

In the presence of Mr. Coulson, Mr. Childs proceeded to operate, assisted by Mr. Goude and Mr. Clarke, after the vapour of chloroform had been administered, so as to bring the patient fully under its influence. A wound through the integuments and superficial fascia, not more than an inch in length, was made on the right side, just beyond the edge of the hernial mass. The finger was directed through the wonnd, and after separating some few bands of cellular and other tissues, which feebly obstructed its course, the neck of the sac was reached, at a depth of at least four inches. The umbilical ring forming the stricture was then felt gripping the neck of the sac, to which it was adherent, by means of some strong bands. These were broken down with the point of the finger, but not without some difficulty, owing to the great quantity of fat and consequent depth of the wound. A director was then insinuated between the sac and the ring, guided by the finger, which still remained in the wound, and the stricture divided. After breaking down some further adhesions, the parts were liberated, and the contents of the sac. immediately returned. The patient had her usual trugs 